\title{
Two High-Order Correct Difference Analogues for the Equation of Multidimensional Heat Flow
}

\author{
By Jim Douglas, Jr., and James E. Gunn
}

1. Introduction. Several high-order accuracy difference equations for the heat equation in one space variable [1] have been proposed, but most do not extend to several space variables with any ease, if at all. Two three-level difference equations are discussed here, each of which is fourth-order correct in space and second in time. One is stable and convergent in $\mathscr{L}_{2}$ for as many as four space variables but is limited essentially to the heat equation itself and in the four space variables case to bounded $r=\Delta t(\Delta x)^{-2}$. The other is stable and convergent in $\mathscr{L}_{2}$ for three space variables and is adapted to extension to more complicated differential equations.

Alternating direction techniques based on the two three-level formulas are developed. These methods retain the accuracy of the original procedures and require much less arithmetic to complete a problem. Only the results will be given here; their analyses will be presented in another paper [3] as examples of a general approach to alternating direction methods.

2. The Difference Equations. It is desired to approximate the $N$-dimensional equation of heat flow

$$
\frac{\partial v}{\partial t}=\Delta v=\sum_{i=1}^{N} \frac{\partial^{2} v}{\partial x_{i}{ }^{2}}
$$

with an implicit difference equation for which the local error is fourth order in the space increment and second order in the time increment. It is known [1] that three or more time levels must be used if the elliptic operator at the advanced time level is to retain a $(2 N+1)$-point form (if $N>1)$. The solution of the $(2 N+1)$-point elliptic equation is itself not simple; however, alternating direction methods may be employed to treat three-level problems of this form without arithmetic complication.

Let $R$ be the cube $0<x_{i}<1, \quad i=1,2, \cdots, N$, and $\partial R$ its boundary. Let $M$ be a positive integer; we impose a square net of size $h=M^{-1}$ upon $R$. It is easily verified that, if $v \in C^{6}$,

$$
\frac{\partial^{2} v_{n}}{\partial x_{i}{ }^{2}}=\frac{1}{3} \Delta_{x_{i}}^{2}\left(v_{n+1}+v_{n}+v_{n-1}\right)-\frac{h^{2}}{12} \frac{\partial^{4} v_{n}}{\partial x_{i}{ }^{4}}+0\left(h^{4}+K^{2}\right)
$$

where $v_{n}=v\left(x_{1}, \cdots x_{N}, n K\right), K$ the time increment, and $\Delta_{x_{i}}^{2}$ is the centered, divided second difference. Thus, if

$$
\begin{gathered}
\Delta_{h}=\sum_{i=1}^{N} \Delta_{x_{i}}^{2} \\
\frac{v_{n+1}-v_{n-1}}{2 K}=\frac{1}{3} \Delta_{h}\left(v_{n+1}+v_{n}+v_{n-1}\right)-\frac{h^{2}}{12} \sum_{i=1} \frac{\partial^{4} v_{n}}{\partial x_{i}{ }^{4}}+0\left(h^{4}+K^{2}\right) .
\end{gathered}
$$

Received April 27, 1962. Research supported in part by the Air Force Office of Scientific Research. 
If we can find a second-order difference approximation to the second term on the right in (2.3), we shall have produced a difference equation locally fourth-order correct in space and second in time. Consider

$$
\Delta^{2} v=\sum_{i=1}^{N} \frac{\partial^{4} v}{\partial x_{i}{ }^{4}}+2 \sum_{i=1}^{N} \sum_{j>i} \frac{\partial^{4} v}{\partial x_{i}{ }^{2} \partial x_{j}{ }^{2}}=\Delta v_{t}=v_{t t} .
$$

Thus, two approaches suggest themselves; we can replace the sum of the fourth derivatives by either

$$
\sum_{i=1}^{N} \frac{\partial^{4} v_{n}}{\partial x_{i}{ }^{4}}=\Delta_{t}{ }^{2} v_{n}-2 \sum_{i=1}^{N} \sum_{j>i}^{N} \Delta_{x_{i}}^{2} \Delta_{x_{j}}^{2} v_{n}+0\left(h^{2}+K^{2}\right)
$$

or

$$
\sum_{i=1}^{N} \frac{\partial^{4} v_{n}}{\partial x_{i}{ }^{4}}=\Delta_{h}\left(\frac{v_{n+1}-v_{n-1}}{2 K}\right)-2 \sum_{i=1}^{N} \sum_{j>i}^{N} \Delta_{x_{i}}^{2} \Delta_{x_{j}}^{2} v_{n}+0\left(h^{2}+K^{2}+\frac{h^{4}}{K}\right) .
$$

We therefore consider two difference analogues to (2.1):

$$
\frac{u_{n+1}-u_{n-1}}{2 K}=\frac{1}{3} \Delta_{h}\left(u_{n+1}+u_{n}+u_{n+1}\right)-\frac{h^{2}}{12}\left(\Delta_{t}^{2} u_{n}-2 \sum_{i=1}^{N} \sum_{j>i}^{N} \Delta_{x_{i}}^{2} \Delta_{x_{j}}^{2} u_{n}\right)
$$

and

$$
\begin{aligned}
\frac{u_{n+1}-u_{n-1}}{2 K}=\frac{1}{3} \Delta_{h}\left(\left(1-\frac{1}{8 r}\right) u_{n+1}+u_{n}+\left(1+\frac{1}{8 r}\right) u_{n-1}\right) & +\frac{h^{2}}{6} \sum_{i=1}^{N} \sum_{j>i}^{N} \Delta_{x_{i}}^{2} \Delta_{x_{j}}^{2} u_{n},
\end{aligned}
$$

where

$$
r=K h^{-2}=\text { constant. }
$$

The choice of constant $r$ is optimum from the point of view of requiring asymptotically the least calculation for a given permissible error [5, Lemma 2], since the error is fourth order in space and second in time. Equation (2.7) is the $N$-dimensional analogue of equation (9.22) of [1].

As both (2.7) and (2.8) involve three time levels, it is necessary to specify both $u_{0}$ and $u_{1}$ to start the calculation. The natural choice for $u_{0}$ is obviously $v_{0}$; the choice of $u_{1}$ will be discussed later.

The equation (2.8) may easily be extended to treat differential equations of the form

$$
\Delta v=a\left(x_{1}, \cdots x_{N}, t\right) \frac{\partial v}{\partial t}+b\left(x_{1}, \cdots x_{N}, t\right) v+c\left(x_{1}, \cdots x_{N}, t\right),
$$

since we need only to approximate the Laplacian of the right hand side [6].

3. Stability and Convergence for (2.7). We consider the difference equation (2.7) on $R$ and compare its solution $u$ with a $C^{6}$ solution $v$ of the boundary value problem for (2.1) on $R$ with

$$
\begin{aligned}
& u_{0}=v_{0} \text { at the net points of } R \\
& u_{n}=v_{n}, n=0,1, \cdots \frac{T}{K}, \text { at the net points of } \partial R .
\end{aligned}
$$


If $e=v-u$, the error equation becomes

$$
\begin{aligned}
& \frac{e_{n+1}-e_{n-1}}{2 K}=\frac{1}{3} \Delta_{h}\left(e_{n+1}+e_{n}+e_{n-1}\right) \\
& \quad-\frac{h^{2}}{12}\left(\Delta_{t}^{2} e_{n}-2 \sum_{i=1}^{N} \sum_{j>i}^{N} \Delta_{x_{i}}^{2} \Delta_{x_{j}}^{2} e_{n}\right)+Q_{n},
\end{aligned}
$$

where $Q_{n}=0\left(h^{4}+K^{2}\right)$. Let us perform a Duhamel decomposition [1] on $e_{n}$. Let

$$
e_{n}=\sum_{m=0}^{n-1} e_{n}^{m}+e_{n}^{*}
$$

where $e_{n}{ }^{m}$ and $e_{n}{ }^{*}=0$ on $\partial R$,

a)

$$
e_{n}^{m}=0, \quad n \leqq m,
$$

b)

$$
\frac{e_{m+1}^{m}}{2 K}=\frac{1}{3} \Delta_{h} e_{m+1}^{m}-\frac{h^{2}}{12 K^{2}} e_{m+1}^{m}+Q_{m}, \quad n=m+1
$$

c) $\frac{e_{n+1}^{m}-e_{n-1}^{m}}{2 K}=\frac{1}{3} \Delta_{h}\left(e_{n+1}^{m}+e_{n}^{m}+e_{n-1}^{m}\right)$

$$
-\frac{h^{2}}{12}\left(\Delta_{t}^{2} e_{n}{ }^{m}-2 \sum_{i=1}^{N} \sum_{j>i} \Delta_{x_{i}}^{2} \Delta_{x_{j}}^{2}{e_{n}}^{m}\right), \quad n \geqq m+1,
$$

and $e_{n}{ }^{*}$ satisfies (3.4c) with $e_{0}{ }^{*}=0, e_{1}^{*}=e_{1}$, and $m=0$. It is necessary to consider $e_{n}^{*}$ in a somewhat different fashion from the others; we shall see, however, that the method of analysis is the same.

Let the grid $\mathfrak{L}_{2}$ norm be

$$
\|u\|=\left(h^{N} \sum_{x \in R}|u|^{2}\right)^{1 / 2},
$$

and let the operator norm be the induced least upper bound norm.

THEOREM 1. The difference equation (2.7) is unconditionally stable with respect to the $\mathfrak{L}_{2}$ norm for $N \leqq 4$; its solution converges to that of (2.1) with an error $\left\|e_{n}\right\|=$ $0\left(h^{4}+K^{2}\right)$ for $N \leqq 4$, provided the solution of $(2.1)$ is $C^{6}$ and $u_{1}$ is approximated to within an error that is $0\left(h^{4}+K^{2}\right)$.

Proof.

Lemma 1.

$$
\left\|e_{m+1}^{m}\right\|=0\left(K\left\|Q_{m}\right\|\right)=0\left(h^{4} K+K^{3}\right)
$$

Proof. From (3.4 b),

$$
\left(1+\frac{h^{2}}{6 K}-\frac{2 K}{3} \Delta_{h}\right) e_{m+1}^{m}=2 K Q_{m}
$$

Hence,

$$
\left\|e_{m+1}^{m}\right\| \leqq 2 K\left\|Q_{m}\right\| \cdot\left\|\left(1+\frac{h^{2}}{6 K}-\frac{2 K}{3} \Delta_{h}\right)^{-1}\right\|
$$

As $\Delta_{h}$ is negative-definite, the indicated inversion is legitimate and

$$
\left\|\left(1+\frac{h^{2}}{12 K^{2}}-\frac{1}{3} \Delta_{h}\right)^{-1}\right\|<1
$$


Lemma 2. Consider a solution of (2.7) with $z_{0}=0, z_{1}$ arbitrary, and $z_{n}=0$ on $\partial R$ for $n=0,1,2, \cdots$. The norm of $z$ is a bounded multiple of $\left\|z_{1}\right\|$ for all $n$, provided that $N \leqq 4$; i.e., (2.7) is stable.

Proof. Let $p=\left(p_{1}, \cdots, p_{N}\right)$, and set $P=\left\{p: p_{j}=1, \cdots, M-1\right\}$. Then, we may expand $z_{n}$ in a sine series:

$$
z_{n}=\sum_{p \in P} \rho_{p}^{(n)} \prod_{j=1}^{N} \sin \pi p_{i} x_{j}
$$

Since

$$
\begin{gathered}
\Delta_{x_{j}}^{2} \sin \pi p_{j} x_{j}=\left(-\frac{4}{h^{2}} \sin ^{2} \frac{\pi p_{j} h}{2}\right) \sin \pi p_{j} x_{j}, \\
\frac{\rho_{p}^{(n+1)}-\rho_{p}^{(n)}}{2 K}=-\frac{1}{3} \sum_{j=1}^{N}\left(-\frac{4}{h^{2}} \sin ^{2} \frac{\pi p_{j} h}{2}\right)\left(\rho_{p}^{(n+1)}+\rho_{p}^{(n)}+\rho_{p}^{(n-1)}\right) \\
-\frac{h^{2}}{12}\left(\frac{\rho_{p}^{(n+1)}-2 \rho_{p}^{(n)}+\rho_{p}^{(n-1)}}{K^{2}}-2 \sum_{i=1}^{N} \sum_{j>i}^{N} \frac{16}{h^{4}} \sin ^{2} \frac{\pi p_{i} h}{2} \sin ^{2} \frac{\pi p_{j} h}{2} \rho_{p}^{(n)}\right) .
\end{gathered}
$$

Let $\sin ^{2}\left(\frac{\pi p_{j} h}{2}\right)=X_{p_{j}}$. Then,

$$
\begin{aligned}
\rho_{p}^{(n+1)}(1+ & \left.\frac{8}{3} r \sum_{j=1}^{N} X_{p_{j}}+\frac{1}{6 r}\right)+\rho_{p}^{(n)}\left(\frac{8}{3} r \sum_{j=1}^{N} X_{p_{j}}-\frac{1}{3 r}\right. \\
& \left.-\frac{16 r}{3} \sum_{i=1}^{N} \sum_{j>i}^{N} X_{p_{i}} X_{p_{j}}\right)+\rho_{p}^{(n-1)}\left(\frac{8}{3} r \sum_{j=1}^{N} X_{p_{j}}-1+\frac{1}{6 r}\right)=0 .
\end{aligned}
$$

Hence,

$$
\rho_{p}^{(n)}=A_{p} \zeta_{1_{p}}^{n}+B_{p} \zeta_{2_{p}}^{n},
$$

where $\zeta_{1_{p}}$ and $\zeta_{2_{p}}$ are the roots of the quadratic equation

$$
\begin{aligned}
f(\zeta)=\left(1+\frac{8 r}{3} \sum_{j=1}^{N} X_{p_{j}}+\frac{1}{6 r}\right) \zeta^{2}+\left(\frac{8 r}{3} \sum_{j=1}^{N} X_{p_{j}}-\frac{1}{3 r}\right. \\
\left.\quad-\frac{16 r}{3} \sum_{i=1}^{N} \sum_{j>i}^{N} X_{p_{i}} X_{p_{j}}\right) \zeta+\left(\frac{8 r}{3} \sum_{j=1}^{N} X_{p_{j}}-1+\frac{1}{6 r}\right)=0 .
\end{aligned}
$$

If the roots of (3.12) are coincident,

$$
\rho_{p}^{(n)}=\left(A_{p}+B_{p} n\right) \zeta_{1 p}^{n} .
$$

Let us ignore the possibility of coincident roots for the moment; we shall see that the analysis will include them as a special case. We note immediately from (3.12) that

$$
\left|\zeta_{1 p} \zeta_{2 p}\right|=\left|\frac{\frac{8 r}{3} \sum_{j} X_{p_{j}}+\frac{1}{6 r}-1}{\frac{8 r}{3} \sum_{j} X_{p_{j}}+\frac{1}{6 r}+1}\right|<1
$$


all $p$, and that

$$
\begin{aligned}
& f(1)=8 r \sum_{j=1}^{N} X_{p_{j}}-\frac{16 r}{3} \sum_{i=1}^{N} \sum_{j>i}^{N} X_{p_{i}} X_{p_{j}} \\
& f(-1)=\frac{2}{3 r}+\frac{8 r}{3} \sum_{j=1}^{N} X_{p_{j}}+\frac{16 r}{3} \sum_{i=1}^{N} \sum_{j>i}^{N} X_{p_{i}} X_{p_{j}}>0, \text { all } p \\
& f^{\prime}(1)=2+8 r \sum_{j=1}^{N} X_{p_{j}}-\frac{16 r}{3} \sum_{i=1}^{N} \sum_{j>i}^{N} X_{p_{i}} X_{p_{j}} \\
& f^{\prime}(-1)=-2-\frac{8 r}{3} \sum_{j=1}^{N} X_{p_{j}}-\frac{1}{2 r}-\frac{16 r}{3} \sum_{i=1}^{N} \sum_{j>i}^{N} X_{p_{i}} X_{p_{j}}<0, \text { all } p .
\end{aligned}
$$

First, let us establish that the roots are not greater than one in magnitude. It is clear that if the roots are complex, the modulus of each is the same and is less than 1 ; thus, we may limit our interest to real roots. In this case, $f(1)$ and $f^{\prime}(1)$ must be nonnegative in order that the roots lie in the unit circle. This places a restriction on the number of space variables we can treat by this high-order correct equation and still maintain unconditional stability. The $X_{p_{i}}$ range from approximately $h^{2} / 4$ to very nearly one. Note that

$$
\frac{16 r}{3} \sum_{i=1}^{N} \sum_{j>i}^{N} X_{p_{i}} X_{p_{j}} \leqq \frac{(N-1)}{2} \frac{16 r}{3} \sum_{i=1}^{N} X_{p_{i}}
$$

and that equality very nearly holds (i.e., to within $0\left(h^{2}\right)$ ) when the $p_{j}$ are all near the upper part of their range. If $f(1)$ is to be nonnegative for all $p$, then

$$
\frac{16}{3} \frac{(N-1)}{2} \leqq 8,
$$

or $N \leqq 4$. This restriction also implies that $f^{\prime}(1)>0$.

From (3.11),

$$
\begin{aligned}
& \rho_{p}^{(0)}=A_{p}+B_{p}, \\
& \rho_{p}^{(1)}=A_{p} \zeta_{1_{p}}+B_{p} \zeta_{2_{p}} .
\end{aligned}
$$

Consequently,

$$
A_{p}=\frac{\rho_{p}^{(1)}}{\zeta_{1_{p}}-\zeta_{2_{p}}}=-B_{p}
$$

If (3.12) has coincident roots, the expression becomes

$$
A_{p}=0, \quad B_{p}=\frac{\rho_{p}^{(1)}}{\zeta_{1_{p}}}
$$

Thus, for distinct roots,

$$
z_{n}=\sum_{p \varepsilon P} \rho_{p}^{(1)} \frac{\zeta_{1_{p}}^{n}-\zeta_{2_{p}}^{n}}{\zeta_{1_{p}}-\zeta_{2_{p}}} \cdot \prod_{j=1}^{N} \sin \pi p_{j} x_{j} .
$$

Let $x^{2}-2 \beta x+\gamma=0$ have distinct roots less than or equal to one in absolute value with their product less than one in absolute value. That this implies that 
$|\beta|<1$ is clear. We shall show that, if $x_{1}, x_{2}$ are the roots, then

$$
\left|\frac{x_{1}{ }^{n}-x_{2}{ }^{n}}{x_{1}-x_{2}}\right| \leqq \frac{2}{1-|\beta|} \text {. }
$$

Let us assume that $\beta>0$ and the roots are real. In this case the root with maximum absolute value is positive and lies between $\beta$ and 1 . We consider two ranges for this root: $\beta<x_{1} \leqq \frac{1}{2}(1+\beta)$ and $\frac{1}{2}(1+\beta)<x_{1}<1$. In the first range,

$$
\left|\frac{x_{1}^{n}-x_{2}{ }^{n}}{x_{1}-x_{2}}=\right| \sum_{k=0}^{n-1} x_{1}^{n-k-1} x_{2}^{k} \mid<\sum_{0}^{\infty} x_{1}^{k}<\frac{2}{1-\beta} \text {. }
$$

If $\frac{1}{2}(1+\beta)<x_{1}<1$, then $x_{2}<\beta-\frac{1}{2}(1-\beta)$, since $x_{1}$ and $x_{2}$ are symmetric with respect to $\beta$, and $\left(x_{1}-x_{2}\right)>1-\beta$. This implies that

$$
\left|\frac{x_{1}{ }^{n}-x_{2}{ }^{n}}{x_{1}-x_{2}}\right|<\frac{\left|x_{1}{ }^{n}\right|+\left|x_{2}\right|^{n}}{1-\beta}<\frac{2}{1-\beta} .
$$

Clearly, $\beta<0$ will produce analogous results. Consider now the case of complex roots with $\beta>0$. Then, $x_{1}=\beta+\epsilon i, \epsilon>0$. Again, consider two ranges for

$$
x_{1}: \beta<\left|x_{1}\right| \leqq \frac{1}{2}(1+\beta) \quad \text { and } \quad \frac{1}{2}(1+\beta)<\left|x_{1}\right|<1 \text {. }
$$

In the first case, the argument is essentially unaltered, and we conclude again that (3.22) holds. If $\frac{1}{2}(1+\beta)<\left|x_{1}\right|<1$, then $\left|x_{1}-x_{2}\right|=2 \epsilon$, and

$$
\epsilon+\beta \geqq|\beta+\epsilon i|>\frac{1+\beta}{2} \text {. }
$$

Thus, $2 \epsilon>1-\beta$ and (3.24) holds without change. If the roots are coincident the pertinent quantity is $\left|n \beta^{n}\right|$, which is also less than $2(1-|\beta|)^{-1}$ for all $n$.

For the quadratic (3.12), the abscissa of the axis is

$$
\beta=\frac{-\frac{8 r}{3} \sum_{j=1}^{N} X_{p_{i}}+\frac{1}{3 r}+\frac{16 r}{3} \sum_{i=1}^{N} \sum_{j>i}^{N} X_{p_{i}} X_{p_{j}}}{2+\frac{16 r}{3} \sum_{j=1}^{N} X_{p_{j}}+\frac{1}{3 r}} .
$$

It is easy to see that

$$
\frac{1}{2}<\beta<\frac{64 r+r^{-1}}{64 r+r^{-1}+6}, \quad N \leqq 4 .
$$

Thus,

$$
\begin{aligned}
\left\|z_{n}\right\| & \leqq \max \left(4, \frac{1}{3}\left(64 r+r^{-1}+6\right)\right)\left\|z_{1}\right\| \\
& =\frac{1}{3}\left(64 r+r^{-1}+6\right)\left\|z_{1}\right\|=C\left\|z_{1}\right\| .
\end{aligned}
$$

It is easily verified that a somewhat stronger inequality holds if the roots are coincident; in any case, (3.28) is valid. This completes the proof of Lemma 2.

The above lemma implies that

$$
\left\|e_{n}{ }^{m}\right\| \leqq C\left\|e_{m+1}^{m}\right\| \leqq 2 C k\left\|Q_{m}\right\| .
$$

Also,

$$
\left\|e_{n}^{*}\right\| \leqq C\left\|e_{1}\right\|
$$


Thus,

$$
\begin{aligned}
\left\|e_{n}\right\| & \leqq \sum_{m=0}^{n-1}\left\|e_{n}^{m}\right\|+\left\|e_{n}^{*}\right\| \geqq \| \\
& \leqq C\left[2 K \sum_{m=0}^{n-1}\left\|Q_{m}\right\|+\left\|e_{1}\right\|\right] \\
& =0\left(h^{4}+K^{2}+\left\|e_{1}\right\|\right) .
\end{aligned}
$$

Therefore, if $\left\|e_{1}\right\|=0\left(h^{4}+K^{2}\right)$, then $\left\|e_{n}\right\|=\theta\left(h^{4}+K^{2}\right)$, and the theorem is demonstrated.

If $N \leqq 3$ (i.e., if the problem arises from physics), then $\beta$ is bounded away from one if we assume merely that $r$ is bounded away from zero. Then, the bound $C$ is independent of the choice of $r$; however, as remarked earlier, constant $r$ is still the optimum choice.

In order to start the method with $u_{1}$ such that $\left\|e_{1}\right\|=0\left(h^{4}+K^{2}\right)$, essentially any of the standard difference equations is sufficient. In particular, the explicit equation

$$
u_{1}=u_{0}+\Delta t \Delta_{h} u_{0}
$$

is adequate.

4. Stability and Convergence for (2.8). Most of the development for this difference equation closely parallels that for (2.7). The argument will be given in outline. The error equation is

$$
\begin{aligned}
\frac{e_{n+1}-e_{n-1}}{2 K}=\frac{1}{3} \Delta_{h}\left(\left(1-\frac{1}{8 r}\right)\right. & \left.e_{n+1}+e_{n}+\left(1+\frac{1}{8 r}\right) e_{n-1}\right) \\
& +\frac{h^{2}}{6} \sum_{i=1}^{N} \sum_{j>i}^{N} \Delta_{x_{i}}^{2} \Delta_{x j}^{2} e_{n}+Q_{n},
\end{aligned}
$$

where $Q_{n}=0\left(h^{4}+K^{2}+h^{6} K^{-1}\right)$. It has the Duhamel decomposition

a)

$$
e_{n}=\sum_{m=0}^{n-1} e_{n}^{m}+e_{n}^{*}
$$

$e_{n}{ }^{m}=0, \quad n \leqq m$,

b) $\quad \frac{e_{m+1}^{m}}{2 K}=\frac{1}{3} \Delta_{h}\left(1-\frac{1}{8 r}\right) e_{m+1}^{m}+Q_{m}, \quad n=m+1$

$$
\text { c) } \begin{aligned}
\frac{e_{n+1}^{m}-e_{n-1}^{m}}{2 K}=\frac{1}{3} \Delta_{h}\left(\left(1-\frac{1}{8 r}\right)\right. & \left.e_{n+1}^{m}+e_{n}{ }^{m}+\left(1+\frac{1}{8 r}\right) e_{n-1}^{m}\right) \\
& +\frac{h^{2}}{6} \sum_{i=1}^{N} \sum_{j>i}^{N} \Delta_{x_{i}}^{2} \Delta_{x_{j}}^{2} e_{n}{ }^{m}, \quad n \geqq m+1,
\end{aligned}
$$

with the same boundary and initial conditions as before.

THEOREM 2. The difference equation (2.8) is $\mathfrak{L}_{2}$-stable for $N \leqq 3$ and convergent with an error that is $0\left(h^{4}+K^{2}\right)$, provided that the solution of $(2.1)$ is $C^{6}$, that $u_{1}$ is estimated with error that is $0\left(h^{4}+K^{2}+h^{6} K^{-1}\right)$, and that $r$ is bounded below away from zero. 
Proof. Lemma 1 carries over in the form

$$
\left\|e_{m+1}^{m}\right\| \leqq \max \left(2 K,(4 r)^{-1} K\right)\left\|Q_{m}\right\| \text {. }
$$

The characteristic equation corresponding to (3.12) is

$$
\begin{aligned}
f(\zeta)=[1+ & \left.\frac{8 r}{3}\left(1-\frac{1}{8 r}\right) \sum_{j=1}^{N} X_{p_{j}}\right] \zeta^{2} \\
+ & {\left[\frac{8 r}{3} \sum_{j=1}^{N} X_{p_{j}}-\frac{16 r}{3} \sum_{i=1}^{N} \sum_{j>i}^{N} X_{p_{i}} X_{p_{j}}\right] \zeta } \\
& \quad+\left[\frac{8 r}{3}\left(1+\frac{1}{8 r}\right) \sum_{j=1}^{N} X_{p_{j}}-1\right]=0 .
\end{aligned}
$$

It is easily verified that $\left|\zeta_{1} \zeta_{2}\right|<1$ and $f(1), f(-1), f^{\prime}(1)$ are positive and $f^{\prime}(-1)$ is negative for $N \leqq 3$. Thus, the roots are less than one in absolute value; the abscissa of the axis of the parabola (4.5) is

$$
\beta=\frac{-8 r \sum_{j=1}^{N} X_{p_{j}}+16 r \sum_{i=1}^{N} \sum_{j>i}^{N} X_{p_{i}} X_{p_{j}}}{6+16 r\left(1-\frac{1}{8 r}\right) \sum_{j=1}^{N} X_{p_{j}}} .
$$

If $N \leqq 3, \beta$ is bounded between $-\frac{1}{2}$ and $+\frac{1}{2}$, so we may proceed exactly as before and find that, for $r$ bounded away from zero,

$$
\left\|e_{n}\right\| \leqq 4\left[\left\|e_{1}\right\|+C K \sum_{m=0}^{n-1}\left\|Q_{m}\right\|\right]=0\left(h^{4}+K^{2}+h^{6} K^{-1}\right)=0\left(h^{4}+K^{2}\right) .
$$

5. Alternating Direction Techniques Associated with (2.7) and (2.8). The authors in a forthcoming paper [3] discuss the application of alternating direction methods to three-level difference equations; the technique may be summarized briefly as follows. First, write the basic difference equation (in this case, either (2.7) or (2.8)) in the form

$$
(I+A) u_{n+1}+B u_{n}+C u_{n-1}=0 .
$$

Decompose $A$ into a sum of simpler operators:

$$
A=\sum_{j=1}^{N} A_{j}
$$

$N$ is frequently, but not necessarily, the dimensionality of the $x$-space. It is necessary that the operator $I+A_{j}$ be invertible. Then, the alternating direction systems become

$$
\begin{gathered}
\left(I+A_{1}\right) w_{n+1}^{(1)}+\left(\sum_{j=2}^{N} A_{j}+B\right) w_{n}+C w_{n-1}=0 \\
\left(I+A_{j}\right) w_{n+1}^{(j)}-w_{n+1}^{(j-1)}-A_{j} w_{n}=0, \quad j=2, \cdots, N \\
w_{n+1}=w_{n+1}^{(N)} .
\end{gathered}
$$


If equation (2.7) for three space variables is rearranged in the form (5.1),

$$
\begin{aligned}
& A=-\frac{2 K}{3} \Delta_{h}+\frac{1}{6 r} I, \\
& B=-\frac{2 K}{3} \Delta_{h}-\frac{1}{3 r} I-\frac{K h^{2}}{3} \sum_{i=1}^{3} \sum_{j>i} \Delta_{x_{i}}^{2} \Delta_{x_{j}}^{2}, \\
& C=-\frac{2 K}{3} \Delta_{h}-\left(1-\frac{1}{6 r}\right) I .
\end{aligned}
$$

One choice for the decomposition of $A$ is

$$
\begin{aligned}
& A_{1}=-\frac{2 K}{3} \Delta_{x_{1}}^{2}+\frac{1}{6 r} I \\
& A_{2}=-\frac{2 K}{3} \Delta_{x_{2}}^{2} \\
& A_{3}=-\frac{2 K}{3} \Delta_{x_{3}}^{2} .
\end{aligned}
$$

It follows from the general results of [3] and a simple calculation for $\beta$ that

$$
\|w-v\|=0\left(h^{4}+K^{2}\right)
$$

for $r$ bounded away from zero. Note that the algebraic equations to be solved at each time step become tridiagonal with the choice (5.5), and, consequently, much less arithmetic is required to evaluate this solution than that of (2.7). As no reduction in the order of the accuracy occurs, the alternating direction analogue possesses a large practical advantage.

For equation (2.8), again for the three space variable case,

$$
\begin{aligned}
& A=-\frac{2 K}{3}\left(1-\frac{1}{8 r}\right) \Delta_{h} \\
& B=-\frac{2 K}{3} \Delta_{h}+\frac{K h^{2}}{3} \sum_{i=1}^{3} \sum_{j>i} \Delta_{x_{i}}^{2} \Delta_{x_{j}}^{2} \\
& C=-\frac{2 K}{3}\left(1+\frac{1}{8 r}\right) \Delta_{h}-I .
\end{aligned}
$$

Let

$$
A_{i}=-\frac{2 K}{3}\left(1-\frac{1}{8 r}\right) \Delta_{x_{i}}^{2}, \quad i=1,2,3 .
$$

Again (5.6) follows for $r \geqq 8^{-1}$.

Both procedures are considered in more detail in [3].

6. Remarks. As was noted in Section 2, the difference equation (2.8) can be modified to treat significantly more complex problems than the heat equation; the general linear self-adjoint differential equation

$$
\nabla \cdot(p \nabla v)=q \frac{\partial v}{\partial t}+r v+m
$$


can be brought into the form (2.9) by a well-known transformation. The technique of treating problems of the type (2.10) is discussed in [6] for one space variable. It is noteworthy that the method of analysis we have used here does not extend either to variable coefficients or to regions more general than a rectangle, the latter because of the noncommutativity of $\Delta_{x_{i}}^{2}$ and $\Delta_{x_{j}}^{2}$ on any but rectangular regions. It is pointed out in [6] that the energy methods used there for problems with variable coefficients on general regions apparently are not sufficiently refined to treat the high-order correct schemes discussed here for more than one space variable. The need for a better method of analysis is thus clearly indicated; an analytical method capable of treating the high-order correct analogue of (2.7) would also have important bearing on alternating direction methods $[3,4,5]$ for problems of the same type, since the residual terms are quite similar.

Rice University

Houston, Texas

California Institute of Technology

Pasadena, California

1. J. Douglas, "A survey of numerical methods for parabolic differential equations," p. 1-54 in Advances in Computers, Vol. II, Academic Press, New York, 1961.

2. J. Douglas, "On the relation between stability and convergence in the numerical solution of linear parabolic and hyperbolic differential equations," J. Soc. Indust. Appl. Math., 4,1956, p. $20-37$.

3. J. Douglas, \& J. E. GunN, "A general formulation of alternating direction methods," (to appear).

4. J. Douglas \& H. H. RACHFoRD, "On the numerical solution of heat conduction problems in two and three space variables," Trans. Amer. Math. Soc., 82, 1956, p. 421-439.

5. J. Douglas, "Alternating direction methods for three space variables," Numer. Math. Vol. 4, 1962, p. 41-43.

6. J. E. GuNN, "Three-level difference methods for parabolic differęntial equations," (to appear). 